\title{
From Aquagels to Aerogels
}

Aerogels, a new class of low-density materials, were discovered early in this century, but they did not attract extensive research interest until the mid-1960s. In the last three decades, a great deal has been accomplished in their synthesis and characterization. Applications of these ultralow-density materials range from extremely efficient thermal insulation and micrometeoroid collectors in the Earth's upper atmosphere to fire-retardants and high-energy particle detectors.

Aerogels are a special class of highly porous foams with ultrafine pore size (less than $1,000 \AA$ ) and an interconnected microstructure with characteristic dimensions less than $100 \AA$. The porosity of the first aerogels in the 1930 s reached $98 \%$ and was pushed all the way to $99.9 \%$ late in 1990 .

The December 1990 issue of the MRS Bulletin described the production methods and material characteristics of many types of low-density microcellular materials. This Historical Note will trace some of the early efforts at developing such substances and the researchers responsible for major developments.

The first aerogels were created by S.S. Kistler at Stanford University in the early 1930s. Normally, when gelatinous substances dry in air, they shrink to about a tenth of their original volume, though they still retain a porosity of about $50 \%$. Kistler found a way to extract fluid from wet gels under high pressure and temperature, drying gels without shrinking them. He named the new solid materials-whose densities ranged from 30 to $300 \mathrm{~kg} / \mathrm{m}^{3}-$ "aerogels."

Initially, Kistler worked with silica to make translucent aerogel slabs. He began with a solution of sodium silicate- "water glass"-mixed with hydrochloric acid. After a day, this mixture would gel, and Kistler added water to wash out the hydrochloric acid. He called the resulting water/silica body an "aquagel." Next, he submerged the aquagel in alcohol, making the next phase, an "alcogel." Washing out the acid and replacing the water with alcohol was a time-consuming business, but it needed to be done because water would dissolve the gel structure under the high temperatures Kistler used in his process.

Normally, when liquid evaporates from a gel, the pores in the gel begin to empty; the increased surface tension creates concave menisci on the walls of the pores. With continued evaporation, the menisci retreat farther into the body of the material, and the surface tension keeps building until eventually the gel body collapses.

\section{The first aerogels were created by S.S. Kistler at Stanford University in the early 1930s.}

Kistler, though, dried his gel solutions in an autoclave, where he could monitor the pressure and temperature. He found that increasing the temperature and pressure above a certain critical threshold turned the gel into a supercritical fluid, where all surface tension vanished and, with it, the menisci that caused the collapse of the gel body. An alcogel made with methanol required a pressure of 81 bars and a temperature of $240^{\circ} \mathrm{C}$ to convert it into an aerogel. With the alcogel sample under the supercritical pressure and temperature conditions, Kistler drained off the fluid from the autoclave, leaving behind a stable "aerogel," which he then brought to room temperature.

At Stanford, Kistler created aerogels out of many materials, including iron oxide, tin oxide, nickel tartrate, alumina, tungsten, even common household materials such as rubber, cellulose, gelatin, and egg white. He also found that the thermal conductivity of his silica aerogel slabs was two orders of magnitude smaller than that of normal silica glass.

Kistler's work went unnoticed for about three decades, until the French government announced an initiative to find new porous materials for storing oxygen and rocket propellants to be used in the budding French space program. A group of researchers under Stanislas J. Teichner at the University of Lyons in France attempted to reproduce Kistler's results from three decades before.

Teichner's team was surprised by how tedious and time-consuming it was just to produce the material-weeks just to produce two small samples! One of Teichner's graduate students, whose thesis was based on the study of aerogels, realized it would take literally years to create enough bulk amounts of aerogels for experimentation, and he suffered a nervous breakdown. By the time he recovered, though, Teichner had introduced an improved aerogel-formation process that took only a day to prepare samples.

Teichner's "sol-gel" process mixed tetramethoxysilane (TMOS) with water. Adding an acid or base catalyst to the mixture caused the formation of silicic acid and methanol. The silicic acid molecules coagulated into a gel body. Altering the $\mathrm{pH}$ and the concentration of the solution affected the density and structure of the resulting gel, which provided another degree of control for the researchers. For many years, the sol-gel method has been the standard process for aerogel research.

Though they were trying to develop a new material for storing rocket fuels, Teichner's group found aerogels useful for detecting Cherenkov radiation in highenergy particle experiments. Cherenkov radiation is given off by high-energy charged particles such as pions, protons, and muons traveling through a medium faster than the speed of light in that medium. Such particles streaming into a detecting medium leave characteristic light trails. Silica aerogels have densities, yielding indices of refraction, that allow detecting Cherenkov radiation from charged particles in an energy range not covered by either liquids or compressed gases. For a typical aerogel, the index of refraction is from 1.002 to 1.3 , which can detect, for example, $30 \mathrm{GeV}$ to $0.8 \mathrm{GeV}$ protons.

At the Deutsches-Elektronen-Synchrotron in Hamburg, Germany, Guinter Poelz and co-workers used Teichner's technique to create 1,700 liters of silica aerogel for such a particle detector. At the University of Lund in Sweden, another group led by Sten Henning and Guy V. Dardel created 1,000 liters of silica aerogel for a similar detector at CERN. The team in Sweden designed a facility with a 3,000 liter autoclave to make $60 \mathrm{~cm}^{2}$ tiles of aerogel. In 1984, though, over 1,000 liters of methanol leaked into the building when the main autoclave gasket failed. An explosion destroyed the entire facility, which took more than four years to rebuild.

Other groups have attempted to find new methods for creating silica aerogels. 
Arlon J. Hunt's team at the University of California at Berkeley replaced the poisonous TMOS with tetraethoxysilane (TEOS) and replaced the methanol portion of the process with $\mathrm{CO}_{2} . \mathrm{CO}_{2}$ is nonexplosive and it needs to be heated to only $31^{\circ} \mathrm{C}$ for drying. The alcohol/ $/ \mathrm{CO}_{2}$ exchange step in Hunt's method, however, takes much longer than Teichner's original technique.

In Ludwigshafen, Germany, a team at BASF sprays an acid and water-glass solution from a mixing jet into a flask, creating aerogel pellets instead of tiles. The pellets are only a few millimeters in diameter, but they are much less expensive to manufacture than aerogel slabs of comparable density $\left(200 \mathrm{~kg} / \mathrm{m}^{3}\right)$ and can still be used for many insulating purposes.

Kistler's original work established the thermal properties of aerogels. In the late 1960s, M. Kaganer in Moscow, working for the All Union Scientific Research Institute of Oxygen, Cryogenic, and Compressor Machinery Construction, provided more detailed analyses of aerogel thermal conductivities. From about 1982 to 1987, Jochen Fricke and his group at the University of Würzburg, Germany, also studied the thermal properties of aerogel materials. One of Fricke's colleagues, Ove Nilsson, discovered that the thermal conductivity of an aerogel changes when the sample is compressed.

Thermal conductivity research has shown that double-paned windows filled with transparent aerogel would insulate three times better than double-paned silver-coated glass filled with argon, which was the best window system previously developed.

Fricke and his group also discovered some interesting acoustical properties of aerogel materials. In 1984, they used a piezoelectric transducer to send pulses of ultrasonic waves through silica aerogel samples. They were surprised to find that the speed of sound was only 100 to $300 \mathrm{~m} / \mathrm{s}$ in the aerogel, while ordinary silica glass transmits sound at $5,000 \mathrm{~m} / \mathrm{s}$. Such low sonic velocities are normally found only in highly compressible materials such as rubber.

Shortly thereafter it was found that the speed of sound decreases further when the aerogel is subjected to mechanical stress-exactly the opposite of the behavior of most solids. With its low density and low speed of sound, an aerogel could be used to improve devices that emit ultrasonic waves to gauge distances, such as auto-focus cameras. An aerogel coating on a ceramic piezoelectric transducer, acting as a buffer between the air and the transducer, could increase the ultrasonic energy emitted by the transducer by as much as a hundred times.

In 1986, Dale W. Schaefer and coworkers at the Sandia National Laboratories in Albuquerque, New Mexico, found that the structure of aerogels was fractal, or self-similar at different scale lengths. In the last several years, other systematic investigations (R. Vacher, T. Woignier, and J. Pelous at the University of Montpellier, and E. Courtens at the IBM Zurich Research Laboratory) studied how aerogel fractality changes with the concentration and $\mathrm{pH}$ of the starting solution. Indeed, much current aerogel work focuses on finding better ways to control the microstructure of aerogels as they form.

Since the resurgence of aerogel work in the past 25 years, much progress has been made. Analysis of the delicate gel body has resulted in intriguing properties with a broad range of possible commercial and research possiblities. When researchers Ian Thomas, Lawrence Hrubesh, and Thomas Tillotson at Lawrence Livermore National Laboratory announced the creation of a new silica aerogel with a porosity of $99.9 \%$, they received over 400 phone calls from various entrepeneurs. Already, the commercial applications suggest not only efficient window insulation and particle detectors, but also high-resolution sound sensors, film industry special effects, fire retardants in buildings, and toy or novelty items. One sign manufacturer wanted to use aerogels as a background material for massive, extremely light signs that would look like letters floating in the sky; an entomologist studying the behavior of borer bees suggested using aerogels as a hive material, which would allow him to observe all their activities inside the hive. No doubt these will be only a fraction of the ways aerogel materials will be used when larger quantities of inexpensive aerogels become available.

KEVIN J. ANDERSON
Advertisers in This Issue

\begin{tabular}{lr}
\hline $\begin{array}{l}\text { Active Materials and } \\
\text { Adaptive Structures }\end{array}$ & 59 \\
\hline AET addax & 8 \\
\hline $\begin{array}{l}\text { Billiton Precursors } \\
\text { B.V. }\end{array}$ & inside back cover \\
\hline Elsevier & 18,65 \\
\hline EPI & 7 \\
\hline
\end{tabular}

High Voltage Engineering Europa B.V. inside

\begin{tabular}{lr}
\hline Huntington Laboratories & 11 \\
\hline Janis Research & 12 \\
\hline Lake Shore Cryotronics & 1 \\
\hline MDC Vacuum Products & 2 \\
\hline MKS Instruments & 33 \\
\hline Optical Associates Inc. & 9 \\
\hline Omicron Associates & 17 \\
\hline Rudolph Research & 16 \\
\hline UHV Instruments & back cover \\
\hline Virginia Semiconductor & 10 \\
\hline Voltaix & 13 \\
\hline
\end{tabular}

For free information about the products and services in this issue, fill out and mail the Reader Service Card, or FAX it to (312) 922-3165.

\title{
SPECIAL BENEFIT FOR MRS MEMBERS...
}

\author{
Your MRS Membership entitles you to a free "Position Wanted" ad in the MRS Bulletin Classified section. \\ Call Mary E. Kaufold at (412) 367-3036 for information.
}

